\title{
Percepciones de padres o acudientes sobre la salud y calidad de vida de sus hijos adolescentes escolarizados
}

The perception of parents and guardians about the health and quality of life of their adolescent children enrolled in school

\section{Percepções de pais ou responsáveis sobre a saúde e a qualidade de vida de seus filhos adolescentes escolarizados}

Mary Luz Jaimes-Valencia, Enf., MSc., PhD. *

Socorro Fajardo-Nates, Enf., Esp., MSc (c) **

John Freddy Arguello, Enf., MSc. ***

Claudia Nathaly Mejía-Arciniegas, Enf. ****

Leidy Carolina Rojas-Arenas, Enf.

Loren Marcela Gallo-Eugenio, Enf.

Nathalia Rocío León-Santos, Est.*******

\section{Resumen}

Introducción. La percepción que tienen los padres o acudientes sobre la calidad de vida de sus hijos, permite realizar una valoración de la salud de los niños teniendo en cuenta las habilidades de participar plenamente en funciones $\mathrm{y}$ actividades físicas, sociales y psicosociales apropiadas para la edad. La investigación en la Calidad de Vida (CV) y calidad de vida relacionada con la salud en niños es un campo que, aunque reciente, ha tenido progresos importantes en los últimos años; para su valoración se han creado varios instrumentos de medición desde la perspectiva cuantitativa, pero son escasos los estudios que analizan las percepciones desde un enfoque cualitativo. Objetivo. Reconocer las

* Enfermera, Magíster en Salud Familiar, Doctora en Enfermería Práctica y Educación. Docente de la Facultad de Ciencias de la Salud, Programa de Enfermería, Universidad Autónoma de Bucaramanga, Floridablanca, Santander, Colombia.

** Enfermera, Especialista en Administración de Servicios de Salud, Candidata a Maestría en E-Learning, Docente de la Facultad de Ciencias de la Salud, Programa de Enfermería, Universidad Autónoma de Bucaramanga, Floridablanca, Santander, Colombia.

*** Enfermero, Magíster en Neurociencias y Biología del Comportamiento. Docente de la Facultad de Ciencias de la Salud, Programa de Enfermería, Universidad Autónoma de Bucaramanga, Floridablanca, Santander, Colombia.

**** Enfermera, Servicio de Hospitalización Fundación Oftalmológica de Santander, Floridablanca, Santander Colombia.

***** Enfermera, Servicio de Hospitalización Clínica Chicamocha, Bucaramanga, Santander, Colombia

****** Enfermera, Servicios de Promoción y Prevención, ESE Hospital Nuestra Señora de los Remedios, San José de Miranda, Santander, Colombia. *******Estudiante VIII Semestre de Enfermería, Miembro del Semillero de investigación Higia, Universidad Autónoma de Bucaramanga, Santander, Colombia.

Correspondencia: Mary Luz Jaimes Valencia, Docente Programa de Enfermería, Universidad Autónoma de Bucaramanga,Floridablanca, Santander, Colombia. E-mail: mjaimes239@unab.edu.co 
Mary Luz Jaimes-Valencia, Socorro Fajardo-Nates, John Freddy Arguello, Claudia Nathaly Mejía-Arciniegas, Leidy Carolina Rojas-Arenas, Loren Marcela Gallo-Eugenio, Nathalia Rocío León-Santos. percepciones que tienen los padres o acudientes sobre la salud y calidad de vida de sus hijos adolescentes. Metodología. Estudio cualitativo con la técnica de grupo focal en la que participaron 9 padres o acudientes. Se realizó un grupo focal, se grabó y transcribió la entrevista. Resultados. Se detectaron 11 categorías que hacen parte de la salud y calidad de vida en la etapa de los adolescentes escolarizados. Surgieron dimensiones que no son reportadas por instrumentos de calidad de vida relacionada con la salud como es la espiritualidad y la tecnología. Respecto a la espiritualidad, estudios han demostrado una correlación positiva fuerte entre los padres, religiosidad y reducción de conductas de riesgo de sus hijos/as. Conclusiones. Abordar a los padres o acudientes permitió identificar otros aspectos de la salud y calidad de vida que pueden afectar a sus hijos en la adolescencia. [Jaimes-Valencia ML, Fajardo-Nates S, Argüello JF, Mejía-Arciniegas CN, Rojas-Arenas LC, GalloEugenio LM, León-Santos NR. Percepciones de padres o acudientes sobre la salud y calidad de vida de sus hijos adolescentes escolarizados. MedUNAB. 2019;21(3):314333. doi: 10.29375/01237047.2736]

Palabras clave: Percepción; Calidad de vida; Bienestar; Salud; Adolescente; Padres.

\section{Abstract}

Introduction. The perception of parents or guardians about their children's quality of life allows to assess the children's health, bearing in mind the abilities to fully participate in age-appropriate physical, social and psychosocial functions and activities. Research on quality of life (QL) and children's quality of life as regards their health is a field that, although recent, has made significant progress in the past years. Various measurement instruments have been developed from a quantitative perspective to assess it, but few studies analyze the perceptions from a qualitative focus. Objective. To recognize the perception of parents and guardians about the health and quality of life of their adolescent children. Methodology. Qualitative study with the focus group technique in which nine parents or caregivers participated. A focus group was created and the interview was recorded and transcribed. Results. Eleven categories that cover the health and quality of life in the stage of adolescents enrolled in school were detected. Dimensions appeared that are not reported by health-related quality of life instruments, such as spirituality and technology. Discussion. In the area of spirituality, studies have demonstrated a strong positive correlation between parents, religiosity and the reduction of risky conducts in their children. Conclusions. Addressing parents or caregivers helped identify other aspects of health and quality of life that can affect their adolescent children. [Jaimes-Valencia ML, Fajardo-Nates S, Argüello JF, Mejía-
Arciniegas CN, Rojas-Arenas LC, Gallo-Eugenio LM, LeónSantos NR. The perception of parents and guardians about the health and quality of life of their adolescent children enrolled in school. MedUNAB. 2019;21(3):314-333. doi: 10.29375/01237047.2736]

Keywords: Perception; Quality of life; Well-being; Health; Adolescents; Parents.

\section{Resumo}

Introdução. A percepção que os pais ou responsáveis têm sobre a qualidade de vida de seus filhos permite uma avaliação da saúde das crianças, considerando as habilidades para participar plenamente de eventos e atividades físicas, sociais e psicossociais adequadas à idade. A pesquisa sobre qualidade de vida (QV) e qualidade de vida relacionada à saúde em crianças é um campo que, apesar de recente, teve importantes avanços nos últimos anos. Para sua avaliação, tem sido criados vários instrumentos de medição da perspectiva quantitativa, mas há poucos estudos que analisam percepções a partir de uma perspectiva qualitativa. Objetivo. Reconhecer as percepções que os pais ou responsáveis têm sobre a saúde e a qualidade de vida de seus filhos adolescentes. Metodologia. Estudo qualitativo com a técnica de grupo focal em que participaram nove pais ou responsáveis. Foi organizado um grupo focal, e a entrevista foi gravada e transcrita. Resultados. Foram detectadas onze categorias que fazem parte da saúde e qualidade de vida na fase de adolescentes escolarizados. Emergiram dimensões que não são relatadas através de instrumentos de qualidade de vida relacionada à saúde, como espiritualidade e tecnologia. Discussão. Em relação à espiritualidade, estudos têm mostrado uma forte correlação positiva entre pais, religiosidade e redução de comportamentos de risco de seus filhos. Conclusões. Dirigirse aos pais ou responsáveis que participaram, ajudou a identificar outros aspectos da saúde e da qualidade de vida que podem afetar seus filhos durante a adolescência. [Jaimes-Valencia ML, FajardoNates S, Argüello JF, Mejía-Arciniegas CN, Rojas-Arenas LC, Gallo-Eugenio LM, León-Santos NR. Percepções de pais ou responsáveis sobre a saúde e a qualidade de vida de seus filhos adolescentes escolarizados. MedUNAB. 2019;21(3):314-333. doi: 10.29375/01237047.2736]

Palavras-chave: Percepção; Qualidade de vida; Bem-estar; Saúde; Adolescentes; Pais

\section{Introducción}

La concepción de la salud ha variado y siempre ha estado íntimamente ligada al proceso de la 
enfermedad, de modo que los conceptos de salud que se han referido, construido y analizado en los diferentes momentos históricos, sustentan la necesidad de los seres humanos de ser vistos como seres biopsicosociales (1). Un referente del concepto de salud es el emitido por la Organización Mundial de la Salud (OMS): como "estado de completo bienestar físico, mental y social, y no sólo la ausencia de enfermedad o dolencia." Además, establece que la salud es un derecho inalienable de todo individuo, independientemente de su raza, religión, ideología y condición sociocultural $(2,3)$.

Conocer el estado de salud de los individuos es estudiar los diferentes determinantes relacionados con la biología de la persona, con el medio ambiente, con el sistema de salud que le atiende y con los estilos de vida que caracterizan su comunidad y, por consiguiente, con su cultura (1). Los estudios sobre salud y enfermedad pueden ser abordados de diversas formas, el Emic o Etic. La visión Emic, permite acceder a información que se encuentra más allá de las apariencias que se pueden observar en las pautas de interacción, las costumbres y las creencias, lo cual permite entender el sentido que estas pueden tener para el sujeto. En contraste, la visión Etic designa la visión del investigador o desde afuera $(2,3)$.

Por otra parte, el desarrollo humano es considerado un proceso complejo que evoluciona por etapas durante el ciclo vital, las cuales van desde el nacimiento hasta la muerte, comprendidas en cuatro dimensiones básicas: desarrollo físico, cognoscitivo, emocional y social. Las capacidades cognoscitivas pueden depender de la salud física y emocional, así como de la experiencia social; el desarrollo social está influenciado por la maduración biológica, la comprensión cognoscitiva y las reacciones emocionales (4). El ciclo vital suele dividirse en tres grandes periodos de desarrollo: infantil, adolescente y adulto. Con respecto a los adolescentes que se encuentran entre los 11 y los 18 años, se dice que generan pensamientos de operaciones formales, formación de una identidad positiva, mayor contacto y sentido de pertenencia con sus pares (5). Los niños y jóvenes son parte importante de nuestra sociedad actual: solo los jóvenes representan un $30 \%$ de la población, por lo cual son claves para el progreso de las regiones; niños y adolescentes con buena salud tienen repercusiones en todos los ámbitos, tanto para el progreso social como económico y político (6).

La percepción de salud depende no solo de las características fisiológicas sino también de experiencias personales, del entorno familiar, del contexto cultural, entre otros; por lo que reconocer condiciones del contexto social y de la vida en comunidad de los individuos puede evitar el impacto de esta en la percepción de salud (7). El estado de salud percibido es uno de los indicadores por excelencia utilizado como aproximación a la situación de salud de las poblaciones en las encuestas de diferentes países. El término auto-percepción del estado de salud, o morbilidad sentida, se refiere a la información suministrada por el individuo acerca de su estado de salud como producto de sus conocimientos e interpretaciones, sin que haya sido confirmado por personal médico. Esta información tiene, en consecuencia, un carácter subjetivo que puede revelar los problemas de salud más comunes en la población a partir de sus experiencias y valores, y no sólo de la demanda de los servicios $(2,3)$.

La Calidad de Vida Relacionada con la Salud (CVRS) es un indicador de salud en crecimiento. Aunque existen diversas definiciones de CVRS, hay consenso en su relación con el concepto de salud autopercibida, en su carácter subjetivo y multidimensional, y en que incluye tanto el funcionamiento positivo como las dimensiones negativas de la salud. La OMS, define la Calidad de Vida (CV) como la "percepción individual de la posición en la vida en el contexto de la cultura y sistema de valores en el cual se vive y su relación con las metas, expectativas, estándares e intereses" (8). Quien usó por primera vez el término "calidad de vida" fue el economista norteamericano John Kenneth Galbraith a finales de los años 50 del siglo XX. Inicialmente, este concepto consistía en el cuidado de la salud personal; luego se convirtió en la preocupación por la salud e higiene pública; se extendió después a los derechos humanos, laborales y ciudadanos; continuó con la capacidad de acceso a los bienes económicos, y finalmente se transformó en la preocupación por la experiencia del sujeto, la vida social, la actividad cotidiana y la propia salud (9).

Otra de las definiciones más utilizada es la de Shumaker \& Naughton, quienes definen CVRS como "la evaluación subjetiva de la influencia del estado de salud, los cuidados sanitarios y la promoción de la salud, sobre la capacidad del individuo para mantener un nivel de funcionamiento que le permite realizar las actividades que le son importantes, y que afectan a su estado general de bienestar..." (8). Por otra parte, KIDSCREEN groups define la CVRS com o un constructo multidimensional que cubre los componentes físicos, emocionales, mentales, sociales y de conducta, del bienestar y de las funciones percibidas por pacientes y/u otros individuos (10). 
Las dimensiones más importantes que incluye la CVRS son: el funcionamiento social, físico y cognitivo; la movilidad y el cuidado personal; y el bienestar emocional. Esta definición parte de la percepción que tiene el individuo sobre su propia salud y es utilizado frecuentemente como sinónimo del concepto de salud auto percibida (8).

La percepción de un estado de salud bueno o muy bueno disminuye con la edad, tanto en hombres como en mujeres; en los jóvenes, las valoraciones positivas de la salud alcanzan casi a nueve de cada diez personas, mientras en los adultos mayores, sólo a cuatro de cada diez. Además, se evidencia que la edad marca diferencias en cuanto a la percepción del estado de salud, pues a medida que esta aumenta, disminuye la proporción de personas que definen su estado de salud como bueno o muy bueno (7).

En un principio, tanto la salud como la calidad de vida en la infancia y la adolescencia eran medidas con indicadores indirectos de los datos epidemiológicos de morbimortalidad. Para ser más concretos, en la última década del siglo XX y en los años posteriores del siglo XXI se comienza a estudiar la CVRS como un concepto holístico, multidimensional e integral, donde por primera vez se tiene en cuenta la percepción del niño sobre su bienestar (11). Se afirma que el desarrollo ha recorrido tres etapas, con un retraso aproximado de diez años en relación con las investigaciones de $\mathrm{CV}$ en adultos. La primera etapa consistió en el desarrollo teórico del constructo $\mathrm{CV}$ en niños como concepto diferente al de los adultos (9). En esta etapa se desarrollaron estudios cualitativos que ayudaron a determinar los componentes del constructo, también se recurrió a estudios que utilizaran la metodología Delphi. La segunda etapa ha estado dedicada a la construcción y desarrollo de instrumentos de medición cuantitativa y la tercera se ha usado en el ámbito clínico (9). La calidad de vida en niños y adolescentes podría definirse entonces como "la percepción del bienestar físico, psicológico y social dentro de un contexto cultural específico de acuerdo a su desarrollo evolutivo y a sus diferencias individuales" (9), mientras que la calidad de vida relacionada con la salud en niños y adolescentes sería la valoración de acuerdo con su desarrollo evolutivo, diferencias individuales y contexto cultural de su funcionamiento físico, psicológico y social cuando este se ha visto limitado o alterado por una enfermedad o accidente (9).

La medida de la CVRS en la infancia y adolescencia puede ser útil para describir la salud de una población y establecer comparaciones entre subgrupos con determinadas características (por ejemplo, sociodemográficas, regionales, etc.), para evaluar el impacto sobre la salud de las intervenciones y las políticas sanitarias, o para focalizar los recursos con relación a las necesidades. En la práctica clínica el estudio de la CVRS puede facilitar el conocimiento del estado de salud y permite identificar niveles de morbilidad, valorar el impacto que tienen los tratamientos sobre la salud individual o grupal, y facilitar el proceso de toma de decisiones clínicas. La utilización de la CVRS infantil como medida de resultado presenta múltiples beneficios tanto en la práctica clínica como en la elaboración de políticas públicas en el campo sanitario. La forma de hacer operativa la medición de la CVRS es a través de instrumentos o cuestionarios estandarizados. Para su correcta utilización, estos deben estar construidos o adaptados a la población que se pretende evaluar, además de concordar con los objetivos de la investigación y presentar propiedades psicométricas adecuadas (8).

Estos instrumentos se aplican como autoinformes y se consideran como el método principal para evaluar la CVRS en los niños una vez que han alcanzado cierta edad y nivel de desarrollo cognitivo; sin embargo, hay numerosas medidas para los proxys o informantes quienes constituyen importantes fuentes complementarias de información sobre la CVRS de niños y adolescentes. Durante la última década se han desarrollado numerosos instrumentos genéricos de CVRS en niños y adolescentes, así como cuestionarios específicos dirigidos a niños con enfermedades crónicas como asma, diabetes, entre otras. En su mayoría, estos instrumentos han sido desarrollados en lenguas extranjeras y son escasos los validados en español. El proceso de validación comprende la adaptación transcultural y la evaluación de las propiedades psicométricas; esto es, determinar la fiabilidad y validez $(10,16)$ (Tabla 1). Es importante reconocer que en la actualidad se cuenta con instrumentos que valoran el constructo de forma cuantitativa proponiendo diferentes dimensiones, pero son escasos los estudios que permiten establecer los componentes que hacen parte de la salud y calidad de vida de los niños y jóvenes con las realidades de los contextos latinoamericanos. De igual manera, son escasos los estudios que valoran estos temas desde la perspectiva de los padres.

Es importante reconocer que los padres cumplen varias funciones en relación con la salud de sus hijos: son los encargados de identificar las necesidades en 
Tabla 1. Instrumentos de valoración de la Calidad de Vida Relacionada con la Salud (CVRS) en niños y adolescentes

\begin{tabular}{|c|c|c|c|c|}
\hline Instrumento & $\begin{array}{l}\text { Creación del } \\
\text { instrumento }\end{array}$ & Rango de edades & Dimensiones & No. Ítems \\
\hline PedsQL (13) & $\begin{array}{l}\text { Estados Unidos } \\
\quad \text { en } 1998\end{array}$ & 2 a 18 años & $\begin{array}{l}\text { Función Física, Función Emocional, } \\
\text { Función Social y Función Escolar. }\end{array}$ & 23 \\
\hline KIDSCREEN (10) & $\begin{array}{l}\text { Desarrollado } \\
\text { simultáneamente } \\
\text { en } 13 \text { países } \\
\text { europeos entre } \\
2001 \text { y } 2004\end{array}$ & 8 a 18 años & $\begin{array}{l}\text { Bienestar Físico, Bienestar } \\
\text { Psicológico, Autonomía, Relación } \\
\text { con los Padres, Amigos, Apoyo } \\
\text { Social y Entorno Escolar }\end{array}$ & $\begin{array}{l}\text { Varía de } \\
10 \text { a } 52\end{array}$ \\
\hline $\begin{array}{l}\text { CHQ (Child Health } \\
\text { Questionnaire) } \\
\text { (14) }\end{array}$ & $\begin{array}{l}\text { Estados Unidos } \\
\quad \text { en } 1996\end{array}$ & 10 a los 18 años & $\begin{array}{l}\text { Función Física, Rol Social-Físico, } \\
\text { Percepción de Salud, Dolor, Rol } \\
\text { Social-Emocional, Rol Social } \\
\text { Comportamiento, Autoestima, Salud } \\
\text { Mental, Comportamiento General, } \\
\text { Actividades Familiares y Cohesión } \\
\text { Familiar }\end{array}$ & $\begin{array}{l}\text { Varía de } \\
28 \text { a } 87\end{array}$ \\
\hline $\begin{array}{c}\text { KINDL } \\
\text { (Fragebogen zur } \\
\text { Lebensqualität } \\
\text { von Kindern and } \\
\text { Judendlichen) (15) }\end{array}$ & $\begin{array}{c}\text { Alemania en } \\
1998\end{array}$ & 3 a 17 años & $\begin{array}{c}\text { Bienestar Físico, Bienestar } \\
\text { Emocional, Actividades Cotidianas } \\
\text { y Bienestar Social. }\end{array}$ & 24 \\
\hline $\operatorname{MOSSF}(16)$ & $\begin{array}{c}\text { Desarrollado en } \\
\text { Estados Unidos } \\
\text { en los inicios de } \\
\text { la década de los } \\
\text { noventa }\end{array}$ & $\begin{array}{l}\text { Adolescentes } \\
\text { mayores de } 13 \\
\text { años }\end{array}$ & $\begin{array}{c}\text { Función Física, Desempeño } \\
\text { Físico, Dolor Corporal, Salud } \\
\text { General, Vitalidad, Función Social, } \\
\text { Desempeño Emocional y Salud } \\
\text { Mental }\end{array}$ & $\begin{array}{l}\text { Varía entre } \\
8 \text { y } 36\end{array}$ \\
\hline $\begin{array}{c}\text { AUQUEI } \\
\text { (Autoquestionnaire } \\
\text { Qualité de } \\
\text { VieEnfant-Imagé) } \\
\text { (11) }\end{array}$ & $\begin{array}{c}\text { Desarrollado } \\
\text { originalmente en } \\
1997\end{array}$ & 4 a 12 años & $\begin{array}{c}\text { Vida familiar, Vida social, } \\
\text { Actividades (colegio y tiempo libre) } \\
\text { y Salud }\end{array}$ & 27 \\
\hline $\begin{array}{l}\text { CHIP (Child } \\
\text { Health and Illness } \\
\text { Profile) (11) }\end{array}$ & $\begin{array}{c}\text { Desarrollado } \\
\text { originalmente en } \\
1993\end{array}$ & 11 a 17 años & $\begin{array}{c}\text { Bienestar, Satisfacción, } \\
\text { Enfermedades, Funciones, } \\
\text { Resistencia y Riesgos }\end{array}$ & 188 \\
\hline $\begin{array}{l}\text { CHIP (Child } \\
\text { Health and Illness } \\
\text { Profile) (11) }\end{array}$ & $\begin{array}{c}\text { Desarrollado } \\
\text { originalmente en } \\
2001\end{array}$ & 6 a 11 años & $\begin{array}{c}\text { Bienestar, Satisfacción, Funciones, } \\
\text { Resistencia y Riesgos }\end{array}$ & 45 \\
\hline EQ-5D-Y (11) & $\begin{array}{c}\text { Desarrollado } \\
\text { originalmente en } \\
2003\end{array}$ & 8 a 18 años & $\begin{array}{l}\text { Movilidad, Autocuidado, } \\
\text { Actividades habituales, Dolor/ } \\
\text { malestar y Ansiedad/depresión }\end{array}$ & 5 \\
\hline $\begin{array}{l}\text { VSP-A (Vécu et } \\
\text { Santé Perçue de } \\
\text { l'Adolescent) (11) }\end{array}$ & $\begin{array}{c}\text { Desarrollado } \\
\text { originalmente en } \\
2000\end{array}$ & 11 a 17 años & $\begin{array}{l}\text { Bienestar psicológico, Energía- } \\
\text { vitalidad, Relaciones con los } \\
\text { amigos/as, Relaciones con los } \\
\text { padres, Tiempo libre/ocio y Colegio }\end{array}$ & 4 \\
\hline
\end{tabular}

Fuente: Elaboración propia de los autores 
salud así como de poner en práctica los tratamientos médicos prescritos. La perspectiva de los padres sobre la salud de sus hijos había contado, hasta hace poco, como el único reporte del estado de salud del niño o adolescente (17). En cuanto a la percepción de la calidad de vida relacionada con la salud, los padres son más optimistas sobre la salud de sus hijos en comparación con las respuestas de estos, pero las percepciones pueden ser distintas dependiendo del estado de salud de su hijo; los padres de niños sanos sobreestiman la calidad de vida, mientras que los de niños enfermos la subestiman. El estado de salud mental del padre afecta la percepción de la CV de su hijo de acuerdo con su propia experiencia, esta percepción también se ve afectada cuando el padre proyecta sus expectativas frente al hijo y lo compara (18). Por lo tanto, resulta de interés desarrollar un estudio de investigación que permita reconocer las percepciones que tienen los padres o acudientes sobre la salud y calidad de vida de sus hijos adolescentes desde un enfoque cualitativo.

\section{Metodología}

\section{Diseño de la investigación}

Estudio descriptivo con enfoque cualitativo. Utilizó la técnica de grupo focal con entrevista grupal semiestructurada; se debe mencionar que esta metodología procura que un grupo de individuos discutan y elaboren, desde la experiencia personal, una temática o hecho social que es objeto de investigación y se recomienda conformar grupos entre 8 y 10 participantes (19).

\section{Participantes}

El grupo focal se conformó teniendo en cuenta a madres, padres y/o acudientes que tuvieran a sus hijos escolarizados en la fase de adolescencia temprana, pertenecientes a una institución educativa pública. Se logró conformar un grupo focal de 9 personas (madre padre y/o acudiente) con hijos adolescentes escolarizados entre los 11 y los 15 años de una institución educativa pública de la ciudad de Bucaramanga (Santander-Colombia).

\section{Criterios de inclusión}

Los criterios de inclusión que se tuvieron en cuenta para la conformación del grupo focal fueron: padres, madres o acudientes de adolescentes entre los 11 y 15 años, de estrato socioeconómico bajo, medio y alto, considerando equilibrio en el sexo de sus hijos, para la conformación del grupo focal.

\section{Criterios de exclusión}

Los criterios de exclusión que se tuvieron en cuenta para la conformación del grupo focal fueron: Padres de adolescentes con patologías crónicas (diabetes, epilepsia, cardiopatías, discapacidades físicas, entre otras) y padres que no acepten de forma voluntaria la participación en el estudio.

\section{Técnica de recolección de datos}

Se organizó un grupo focal compuesto por 9 participantes, madres y padres de familia con hijos de sexo femenino y masculino, de adolescentes escolarizados entre los 11 y 15 años, a quienes se les invitó a participar del estudio y aceptaron participar en el grupo focal. En este, se le presentaron 10 preguntas semiestructuradas a los participantes. El tiempo promedio de la entrevista en el grupo focal fue de 45 minutos.

\section{Procedimiento}

Con previa aprobación por parte de las directivas de la institución educativa y haciendo uso de una circular en la cual se describió en forma detallada el propósito del estudio de investigación, se realizó un contacto inicial vía telefónica con 20 padres de familia para información y sensibilización de los padres con respecto a la propuesta de investigación, estableciendo aspectos éticos correspondientes de participación voluntaria y confidencialidad, estipulados en la firma del consentimiento informado. Del total de madres y padres de familia citados acudieron 9 personas con las que se realizó la firma del consentimiento informado y se procedió a realizar el grupo focal.

Se contó con dos de los investigadores como agentes facilitadores para el grupo focal quienes se encargaron de aplicar la entrevista semiestructurada de 10 preguntas abiertas que permitieron establecer las percepciones de los padres de familia sobre la salud y calidad de vida de sus hijos. Otros dos investigadores como observadores quienes tomaron nota de la actividad desarrollada a través de un diario de campo. La información fue recolectada a través de técnicas de grabación. La entrevista se transcribió y se realizó análisis de contenido que 


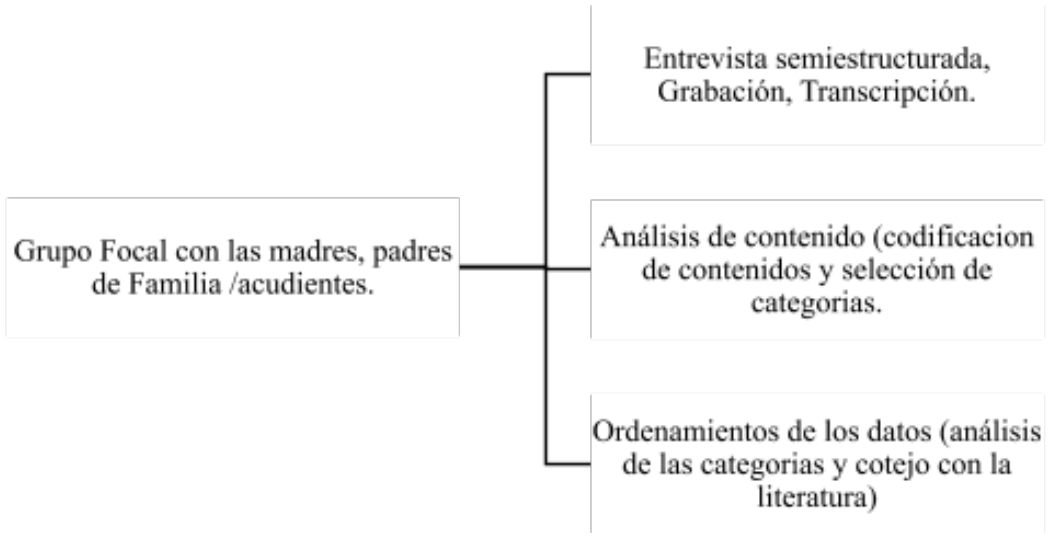

Figura 1. Organización de la Información

Fuente: Elaboración propia de los autores

\begin{tabular}{|c|c|c|c|c|}
\hline & & $\begin{array}{l}\text { Análisis de } \\
\text { contenido }\end{array}$ & & \\
\hline Г & 1 & & $T$ & 7 \\
\hline $\begin{array}{l}\text { 1. Lectura lineal } \\
\text { (codificacion de } \\
\text { contenidos) }\end{array}$ & $\begin{array}{l}\text { 2. Agrupacion de } \\
\text { la codificacion } \\
\text { de contenidos } \\
\text { con cada } \\
\text { pregunta y } \\
\text { lectura entre } \\
\text { lineas }\end{array}$ & $\begin{array}{c}\text { 3. Primeras } \\
\text { categorias y } \\
\text { subcategorias } \\
\text { (propuestas por } \\
\text { los } \\
\text { investigadores) }\end{array}$ & $\begin{array}{l}\text { 4. Reagrupacion } \\
\text { de categorias y } \\
\text { subcategorias }\end{array}$ & $\begin{array}{l}\text { 5. Categorias } \\
\text { definitivas }\end{array}$ \\
\hline
\end{tabular}

Figura 2. Análisis de Contenido

Fuente: Elaboración propia de los autores

permitió la organización de categorías y subcategorías.

\section{Organización de la información}

La organización de la información del estudio cualitativo cumplió la siguiente estructura (Figura 1).

Una vez obtenida la información de los participantes, se procedió a transcribir la entrevista grabada. Con el material transcrito, se realizó el análisis de contenido (Figura 2) a través de la lectura lineal como primer paso, allí se fraccionaron los diferentes apartados para la creación de codificación de contenido (Tabla 2) que permitió identificar las respuestas de los sujetos entrevistados. En un segundo paso de organización de la información, se realizó una segunda lectura para agrupar las diferentes respuestas codificadas con cada pregunta de la entrevista. En un tercer paso, se generaron categorías y subcategorías preliminares con codificación, propuestas por los investigadores con base en la información de la literatura, sin alejar la categoría del parafraseo de los sujetos investigados, además, fue necesario realizar una lectura entre líneas para algunas respuestas codificadas para lograr ubicarlo en las categorías. Finalmente, se reagruparon las diferentes categorías y subcategorías, quedando establecidas 11 categorías, y con estos los investigadores cotejaron la información de los sujetos entrevistados con los diferentes estudios de la literatura. 
Tabla 2. Creación de codificación de contenido

\begin{tabular}{|c|c|}
\hline CÓDIGO & INTERPRETACIÓN \\
\hline P1M1INT1 & Pregunta 1 Mamá 1 Intervención 1 \\
\hline P1M2INT1 & Pregunta 1 Mamá 2 Intervención 1 \\
\hline P1M2INT2 & Pregunta 1 Mamá 2 Intervención 2 \\
\hline P1M2INT3 & Pregunta 1 Mamá 2 Intervención 3 \\
\hline P1M2INT4 & Pregunta 1 Mamá 2 Intervención 4 \\
\hline P1M2INT5 & Pregunta 1 Mamá 2 Intervención 5 \\
\hline P1M2INT6 & Pregunta 1 Mamá 2 Intervención 6 \\
\hline P1M7INT1 & Pregunta 1 Mamá 7 Intervención 1 \\
\hline P1M7INT2 & Pregunta 1 Mamá 7 Intervención 2 \\
\hline P1M7INT3 & Pregunta 1 Mamá 7 Intervención 3 \\
\hline P1M7INT4 & Pregunta 1 Mamá 7 Intervención 4 \\
\hline P1P1INT1 & Pregunta 1 Papá 1 Intervención 1 \\
\hline P1A1INT2 & $\begin{array}{c}\text { Pregunta } 1 \text { Abuelo } 1 \text { Intervención } \\
2\end{array}$ \\
\hline P1A1INT4 & $\begin{array}{c}\text { Pregunta } 1 \text { Abuelo } 1 \text { Intervención } \\
4\end{array}$ \\
\hline P2M1INT2 & Pregunta 2 Mamá 1 Intervención 2 \\
\hline P2P1INT2 & Pregunta 2 Papá 1 Intervención 2 \\
\hline P2A1INT5 & Pregunta 2 Abuelo 1 Intervención \\
\hline P3M1INT3 & Pregunta 3 Mamá 1 Intervención 3 \\
\hline P3M2INT7 & Pregunta 3 Mamá 2 Intervención 7 \\
\hline P3M5INT4 & Pregunta 3 Mamá 5 Intervención 4 \\
\hline P3M6INT1 & Pregunta 3 Mamá 6 Intervención 1 \\
\hline P3P1INT4 & Pregunta 3 Papá 1 Intervención 1 \\
\hline
\end{tabular}

\begin{tabular}{|c|c|}
\hline P4M7INT1 & Pregunta 4 Mamá 7 Intervención 1 \\
\hline P4P1INT5 & Pregunta 4 Papá 1 Intervención 5 \\
\hline P5M1INT6 & Pregunta 5 Mamá 1 Intervención 6 \\
\hline P5M1INT7 & Pregunta 5 Mamá 1 Intervención 7 \\
\hline P5M2INT9 & Pregunta 5 Mamá 2 Intervención 9 \\
\hline P5M3INT1 & Pregunta 5 Mamá 3 Intervención 1 \\
\hline P5M3INT2 & Pregunta 5 Mamá 3 Intervención 2 \\
\hline P5M3INT3 & Pregunta 5 Mamá 3 Intervención 3 \\
\hline P5M3INT4 & Pregunta 5 Mamá 3 Intervención 4 \\
\hline P5M6INT2 & Pregunta 5 Mamá 6 Intervención 2 \\
\hline P5M6INT3 & Pregunta 5 Mamá 6 Intervención 3 \\
\hline P5M7INT1 & Pregunta 5 Mamá 7 Intervención 1 \\
\hline P5P1INT7 & Pregunta 5 Papá 1 Intervención 7 \\
\hline P5P1INT8 & Pregunta 5 Papá 1 Intervención 8 \\
\hline P5P1INT9 & Pregunta 5 Papá 1 Intervención 9 \\
\hline P5P1INT10 & Pregunta 5 Papá 1 Intervención 10 \\
\hline P5P1INT12 & Pregunta 5 Papá 1 Intervención 12 \\
\hline P5P1INT13 & Pregunta 5 Papá 1 Intervención 13 \\
\hline P5A1INT3 & $\begin{array}{c}\text { Pregunta } 5 \text { Abuelo } 1 \text { Intervención } \\
3\end{array}$ \\
\hline P5A1INT6 & $\begin{array}{c}\text { Pregunta } 5 \text { Abuelo } 1 \text { Intervención } \\
6\end{array}$ \\
\hline P6M1INT8 & Pregunta 6 Mamá 1 Intervención 8 \\
\hline P6M4INT2 & Pregunta 6 Mamá 4 Intervención 2 \\
\hline P7M1INT1 & Pregunta 7 Mamá 1 Intervención 1 \\
\hline P7M1INT3 & Pregunta 7 Mamá 1 Intervención 3 \\
\hline P7M1INT4 & Pregunta 7 Mamá 1 Intervención 4 \\
\hline
\end{tabular}


Percepciones de padres o acudientes sobre la salud y calidad de vida de sus hijos adolescentes escolarizados

\begin{tabular}{|c|c|}
\hline P7M1INT5 & Pregunta 7 Mamá 1 Intervención 5 \\
\hline P7M1INT9 & Pregunta 7 Mamá 1 Intervención 9 \\
\hline P7M2INT1 & Pregunta 7 Mamá 2 Intervención 1 \\
\hline P7M2INT8 & Pregunta 7 Mamá 2 Intervención 8 \\
\hline P7M3INT4 & Pregunta 7 Mamá 3 Intervención 4 \\
\hline P7M4INT2 & Pregunta 7 Mamá 4 Intervención 2 \\
\hline P7M5INT1 & Pregunta 7 Mamá 5 Intervención 1 \\
\hline P7M5INT5 & Pregunta 7 Mamá 5 Intervención 5 \\
\hline P7M6INT4 & Pregunta 7 Mamá 6 Intervención 4 \\
\hline P7M7INT1 & Pregunta 7 Mamá 7 Intervención 1 \\
\hline P7M7INT2 & Pregunta 7 Mamá 7 Intervención 2 \\
\hline P7P1INT5 & Pregunta 7 Papá 1 Intervención 5 \\
\hline P7P1INT11 & Pregunta 7 Papá 1 Intervención 11 \\
\hline P7P1INT14 & Pregunta 7 Papá 1 Intervención 14 \\
\hline P7P1INT17 & Pregunta 7 Papá 1 Intervención 17 \\
\hline P7P1INT18 & Pregunta 7 Papá 1 Intervención 18 \\
\hline P7P1INT19 & Pregunta 7 Papá 1 Intervención 19 \\
\hline P7A1INT3 & $\begin{array}{c}\text { Pregunta } 7 \text { Abuelo } 1 \text { Intervención } \\
3\end{array}$ \\
\hline P7A1INT7 & $\begin{array}{c}\text { Pregunta } 7 \text { Abuelo } 1 \text { Intervención } \\
7\end{array}$ \\
\hline P8M2INT2 & Pregunta 8 Mamá 2 Intervención 2 \\
\hline P8M3INT6 & Pregunta 8 Mamá 3 Intervención 6 \\
\hline P8M4INT2 & Pregunta 8 Mamá 4 Intervención 2 \\
\hline P8M6INT1 & Pregunta 8 Mamá 6 Intervención 1 \\
\hline P8A1INT1 & $\begin{array}{c}\text { Pregunta } 8 \text { Abuelo } 1 \text { Intervención } \\
1\end{array}$ \\
\hline P9M1INT10 & Pregunta 9 Mamá 1 Intervención \\
\hline
\end{tabular}

\begin{tabular}{|c|c|}
\hline P9M2INT6 & Pregunta 9 Mamá 2 Intervención 6 \\
\hline P9M3INT5 & Pregunta 9 Mamá 3 Intervención 5 \\
\hline P9M6INT1 & Pregunta 9 Mamá 6 Intervención 1 \\
\hline P9P1INT5 & Pregunta 9 Papá 1 Intervención 5 \\
\hline P9P1INT6 & Pregunta 9 Papá 1 Intervención 6 \\
\hline P9P1INT16 & Pregunta 9 Papá 1 Intervención 16 \\
\hline P9P1NT17 & Pregunta 9 Papá 1 Intervención 17 \\
\hline P9P1INT19 & Pregunta 9 Papá 1 Intervención 19 \\
\hline P10M4INT4 & Pregunta 10 Mamá 4 Intervención \\
\hline P10M5INT2 & $\begin{array}{c}\text { Pregunta 10 Mamá 5 Intervención } \\
2\end{array}$ \\
\hline P10M5INT3 & $\begin{array}{c}\text { Pregunta 10 Mamá 5 Intervención } \\
3\end{array}$ \\
\hline P10P1INT20 & $\begin{array}{r}\text { Pregunta 10 Papá 1 Intervención } \\
\text { 20 }\end{array}$ \\
\hline
\end{tabular}

Fuente: Elaboración propia de los autores

\section{Validez del Estudio}

Respecto a los criterios de validez se tuvo en cuenta los siguientes aspectos:

- Saturación de los datos, es decir, la mayoría de las categorías tales como: relaciones con los padres y vida familiar, autonomía, tiempo libre y ocio, lograron obtener la mayor cantidad de datos y por ende mayor saturación de información.

- Credibilidad determinada por las narrativas contadas por los sujetos en las diferentes categorías teniendo en cuenta sus experiencias vividas como padres de hijos adolescentes.

- Confirmabilidad: se realizaron grabaciones y se transcribió textualmente la entrevista grupal, posteriormente, se llevó a cabo la respectiva codificación para seleccionar las diferentes categorías definitivas, finalmente se cotejó con la literatura. 


\section{Resultados}

\section{Resultados de las características sociodemográficas de los participantes}

Respecto a las características sociodemográficas de los participantes del grupo focal, se destaca que todos son procedentes de la ciudad de Bucaramanga. Se contó con la participación de 2 padres de familia de sexo masculino que representan el $22.22 \%$ y 7 madres de familia que representan el $77.77 \%$. Dentro de las características de los hijos se encontró que son individuos escolarizados entre los 11 y 15 años, 5 son hombres y 4 mujeres. Son chico escolarizados quienes se encuentran cursando entre sexto y octavo grado. De otra parte, el $55.55 \%$ de los hijos pertenecen a estratos socioeconómicos bajos (Tabla 3).

Tabla 3. Características sociodemográficas de los participantes del estudio del grupo focal sobre la percepción que tienen los padres o acudientes acerca de la salud y calidad de vida de sus hijos adolescentes.

\begin{tabular}{|c|c|c|}
\hline Variable & $\mathbf{N}$ & $\%$ \\
\hline \multicolumn{3}{|l|}{ Características de los padres } \\
\hline \multicolumn{3}{|l|}{ Sexo } \\
\hline Hombre & 2 & 22.22 \\
\hline Mujeres & 7 & 77.77 \\
\hline escolarizados & Características de los adolescentes & \\
\hline \multicolumn{3}{|l|}{ Sexo } \\
\hline Hombre & 5 & 55.5 \\
\hline Mujeres & 4 & 44.44 \\
\hline \multicolumn{3}{|c|}{ Edad los adolescentes escolarizados } \\
\hline 11-15 años & 9 & 100 \\
\hline \multicolumn{3}{|c|}{ Nivel de escolaridad de los adolescentes } \\
\hline Secundaria (6 a 8 nivel) & 9 & 100 \\
\hline $\begin{array}{l}\text { Estrato socioeconómico de la res } \\
\text { del adolescente escolarizado rep } \\
\text { por el padre de familia }\end{array}$ & & \\
\hline Estratos bajos (1 y 2) & 5 & 55.55 \\
\hline Estratos medios ( 3 y 4$)$ & 3 & 33.33 \\
\hline Estratos altos (5 y 6$)$ & 1 & 11.11 \\
\hline
\end{tabular}

Fuente: Elaboración propia de los autores 


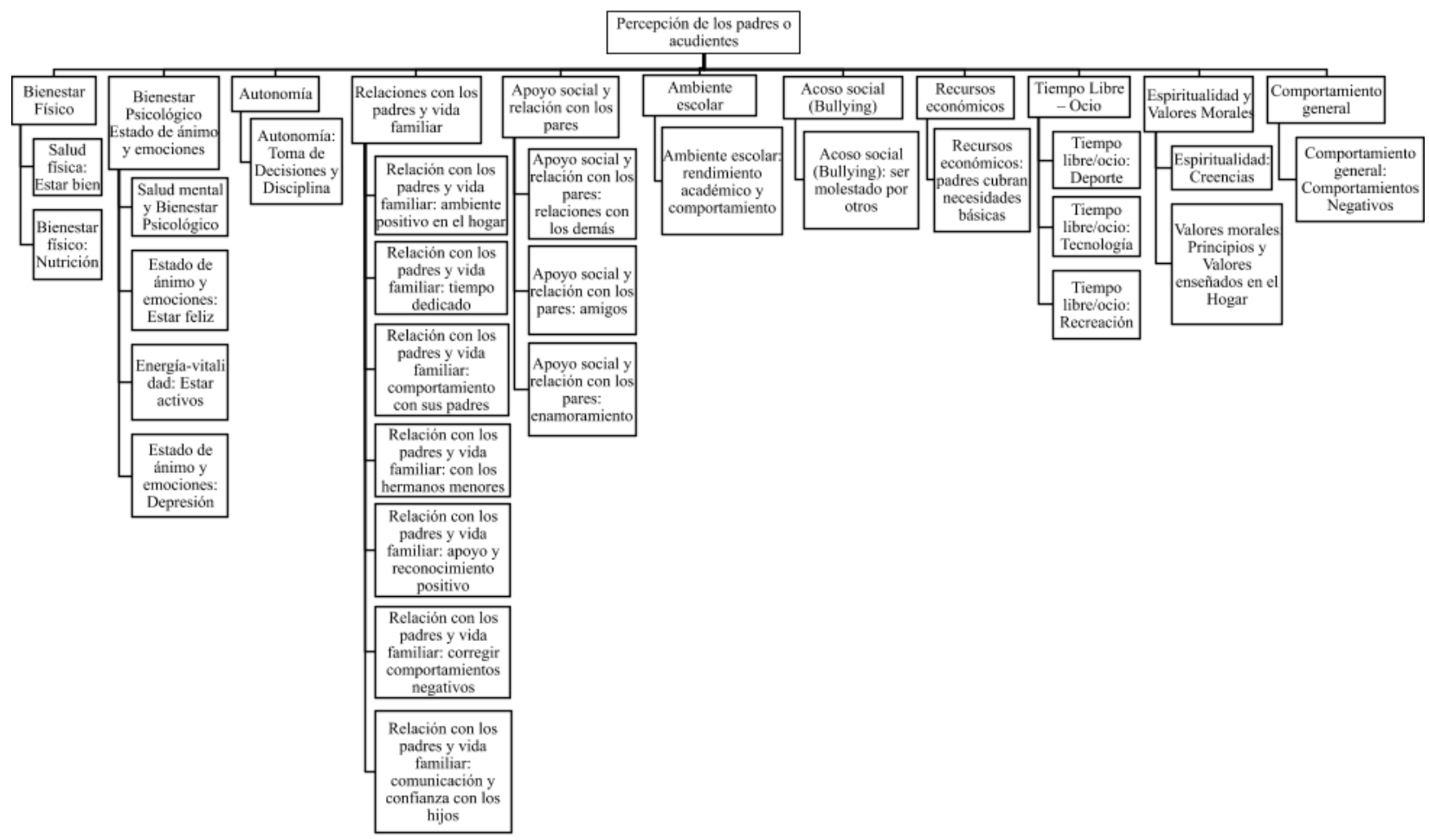

Figura 3. Categorías y subcategorías definitivas de la percepción de padres y acudientes sobre Calidad de Vida Relacionada con la Salud CVRS de sus hijos adolescentes

Fuente:Elaboración propia de los autores

\section{Resultados de las categorías definitivas}

Respecto a la definición de las categorías para el estudio es importante destacar que fue necesario realizar un análisis detallado de las respuestas dadas por los participantes que permitiera darle sentido a cada una de las subcategorías que conforman las categorías, a continuación, se presentan las categorías definitivas y la relevancia que se tuvo en cuenta para la creación de estas (Tabla 4).

En este se identificaron 13 categorías y 36 subcategorías preliminares con codificación, de las cuales, finalmente, se distinguieron 11 categorías definitivas (Tabla 4 y 5) (Figura 3) desde la perspectiva de los padres o acudientes. Las categorías o dimensiones más relevantes de este estudio corresponden a: relaciones con los padres y vida familiar, autonomía, tiempo libre y ocio, destacándose el uso inadecuado de la tecnología como un aspecto negativo que afecta la salud y calidad de vida, el apoyo social y relaciones con los pares. Por otra parte, cabe destacar que los padres identifican la espiritualidad y valores morales como componente de la salud y calidad de vida en los niños y adolescentes.

\section{Discusión}

Las narrativas de los padres permitieron construir las categorías definitivas, las cuales se logran contrastar con otros estudios como se presenta a continuación:

\section{Bienestar físico}

En la infancia, unas pautas alimentarias saludables pueden prevenir enfermedades relacionadas con la alimentación, como la obesidad. Los progenitores influyen en el contexto alimentario infantil con modelos, a menudo, permisivos con la elección de la alimentación de sus hijos y en aspectos tales como el tipo, la calidad, la cantidad y los horarios en que se lleva a cabo (28). Por lo anterior, algunas de las narrativas de los padres expresan lo siguiente:

"Una buena alimentación buena sana, bien balanceada, 
Tabla 4. Categorías y subcategorías definitivas con codificación

\begin{tabular}{|c|c|c|}
\hline $\begin{array}{l}\text { Categorías } \\
\text { Definitivas }\end{array}$ & Subcategorías Definitivas & Códigos \\
\hline \multirow[b]{2}{*}{ Bienestar Físico } & Salud física: Estar bien & P1M7INT1 \\
\hline & Bienestar físico: Nutrición & $\begin{array}{c}\text { P1M2INT4, P1A1INT2, } \\
\text { P5A1INT3, P7A1INT3, } \\
\text { P8A1INT1 }\end{array}$ \\
\hline \multirow{4}{*}{$\begin{array}{c}\text { Bienestar } \\
\text { Psicológico: } \\
\text { Estado de ánimo y } \\
\text { emociones }\end{array}$} & Salud mental y Bienestar Psicológico & $\begin{array}{l}\text { P1A2INT4, P5M7INT1, } \\
\text { P1M1INT1 }\end{array}$ \\
\hline & Estado de ánimo y emociones: Estar feliz & $\begin{array}{c}\text { P1M2INT1, P2A1INT5, } \\
\text { P3M2INT7, P9M2INT6, } \\
\text { P9P1INT19 }\end{array}$ \\
\hline & Energía-vitalidad: Estar activos & P3M2INT7 \\
\hline & Estado de ánimo y emociones: Depresión & $\begin{array}{l}\text { P5P1INT13, P5M1INT6, } \\
\text { P5M1INT7 }\end{array}$ \\
\hline Autonomía & $\begin{array}{l}\text { Autonomía: Toma de Decisiones y } \\
\text { Disciplina }\end{array}$ & $\begin{array}{c}\text { P4P1INT15, P7M4INT2, } \\
\text { P9P1INT5, P9M6INT1, } \\
\text { P9P1INT6, P4M7INT1, } \\
\text { P7M7INT1 }\end{array}$ \\
\hline \multirow{7}{*}{$\begin{array}{l}\text { Relaciones con } \\
\text { los padres y vida } \\
\text { familiar }\end{array}$} & $\begin{array}{l}\text { Relación con los padres y vida familiar: } \\
\text { ambiente positivo en el hogar }\end{array}$ & $\begin{array}{l}\text { P1M2INT2, P7M7INT2, } \\
\text { P7M1INT3 }\end{array}$ \\
\hline & $\begin{array}{l}\text { Relación con los padres y vida familiar: } \\
\text { tiempo dedicado }\end{array}$ & $\begin{array}{c}\text { P1M2INT6, P6M4INT2, } \\
\text { P8M6INT1, P8M3INT6, } \\
\text { P5M3INT1 }\end{array}$ \\
\hline & $\begin{array}{l}\text { Relación con los padres y vida familiar: } \\
\text { comportamiento con sus padres }\end{array}$ & $\begin{array}{c}\text { P2A1INT5, P2P1INT2, } \\
\text { P2M1INT2, P9P1INT19, } \\
\text { P9P1INT19 }\end{array}$ \\
\hline & $\begin{array}{l}\text { Relación con los padres y vida familiar: } \\
\text { con los hermanos menores }\end{array}$ & $\begin{array}{l}\text { P5P1INT7, P5M6INT2, } \\
\text { P5M6INT2, P5P1INT8, } \\
\text { P5A1INT6, P5P1INT8 }\end{array}$ \\
\hline & $\begin{array}{l}\text { Relación con los padres y vida familiar: } \\
\text { apoyo y reconocimiento positivo }\end{array}$ & $\begin{array}{l}\text { P7M1INT1, P7M1INT1, } \\
\text { P7M1INT4, P7M5INT5, } \\
\text { P7M2INT8, P7M1INT5 }\end{array}$ \\
\hline & $\begin{array}{l}\text { Relación con los padres y vida familiar: } \\
\text { corregir comportamientos negativos }\end{array}$ & $\begin{array}{l}\text { P7P1INT5, P7M3INT4, } \\
\text { P7P1INT18, P7M5INT1 }\end{array}$ \\
\hline & $\begin{array}{l}\text { Relación con los padres y vida familiar: } \\
\text { comunicación y confianza con los hijos }\end{array}$ & $\begin{array}{c}\text { P7P1INT17, P7M4INT1, } \\
\text { P7P1INT14, P7M4INT2, } \\
\text { P9P1INT16, P9P1NT17, } \\
\text { P7M4INT2 }\end{array}$ \\
\hline
\end{tabular}


Percepciones de padres o acudientes sobre la salud y calidad de vida de sus hijos adolescentes escolarizados

\begin{tabular}{|c|c|c|}
\hline \multirow{3}{*}{$\begin{array}{l}\text { Apoyo social y } \\
\text { relación con los } \\
\text { pares }\end{array}$} & $\begin{array}{l}\text { Apoyo social y relación con los pares: } \\
\text { relaciones con los demás }\end{array}$ & $\begin{array}{l}\text { P3M6INT1, P4M7INT1, } \\
\text { P1M7INT1, P3P1INT4 }\end{array}$ \\
\hline & $\begin{array}{l}\text { Apoyo social y relación con los pares: } \\
\text { amigos }\end{array}$ & P8M4INT2, P5M3INT4 \\
\hline & $\begin{array}{l}\text { Apoyo social y relación con los pares: } \\
\text { enamoramiento }\end{array}$ & P5P1INT12 \\
\hline Ambiente escolar & $\begin{array}{l}\text { Ambiente escolar: rendimiento académico } \\
\text { y comportamiento }\end{array}$ & P2M1INT2, P3M1INT3 \\
\hline $\begin{array}{l}\text { Aceptación social } \\
\text { (Bullying) }\end{array}$ & $\begin{array}{l}\text { Aceptación social (Bullying): } \\
\text { molestado por otros }\end{array}$ & P7M4INT2 \\
\hline $\begin{array}{l}\text { Recursos } \\
\text { económicos }\end{array}$ & $\begin{array}{l}\text { Recursos económicos: padres cubran } \\
\text { necesidades básicas }\end{array}$ & $\begin{array}{c}\text { P1M7INT1, P6M1INT8, } \\
\text { P7M1INT9, P7M5INT1, } \\
\text { P7A1INT7 }\end{array}$ \\
\hline \multirow{3}{*}{$\begin{array}{l}\text { Tiempo Libre - } \\
\text { Ocio }\end{array}$} & Tiempo libre/ocio: Deporte & P1M7INT4 \\
\hline & Tiempo libre/ocio: Tecnología & $\begin{array}{l}\text { P5M6INT3, P5M2INT9, } \\
\text { P5P1INT9, P5P1INT10, } \\
\text { P5M3INT2, P5M3INT3, } \\
\text { P7P1INT11, P7P1INT18 }\end{array}$ \\
\hline & Tiempo libre/ocio: Recreación & P7A1INT7 \\
\hline \multirow{2}{*}{$\begin{array}{l}\text { Espiritualidad y } \\
\text { Valores Morales }\end{array}$} & Espiritualidad: Creencias & $\begin{array}{l}\text { P1M2INT3, P1M1INT1, } \\
\text { P8M2INT2, P9M3INT5 }\end{array}$ \\
\hline & $\begin{array}{l}\text { Valores morales: Principios y Valores } \\
\text { enseñados en el Hogar }\end{array}$ & $\begin{array}{l}\text { P1P1INT1, P7M6INT4, } \\
\text { P7P1INT19 }\end{array}$ \\
\hline $\begin{array}{l}\text { Comportamiento } \\
\text { general }\end{array}$ & $\begin{array}{l}\text { Comportamiento general: } \\
\text { Comportamientos Negativos }\end{array}$ & P3M5INT4, P3M6INT1 \\
\hline
\end{tabular}

Fuente: Elaboración propia de los autores

frutas verduras una comida sana tienen a la persona con una muy buena salud, con alimentación, con eso tienen buena salud" (P8A1INT1).

\section{Bienestar psicológico, estado de ánimo y emociones}

El bienestar psicológico, estado de ánimo y las emociones determinadas por la calidad de las relaciones entre padres e hijos se asocia significativamente con la salud psicológica y el desarrollo infantil, incluido el aprendizaje y las habilidades sociales, la salud mental y los comportamientos relacionados con la salud, y siguen influyendo en el desarrollo de conductas saludables y habilidades sociales en la edad adulta (22). Cabe resaltar, que los padres tienen un papel clave en el desarrollo de actitudes sirviendo de modelo a sus hijos, pues las interacciones emocionales con el cuidador, además de fomentar el desarrollo emocional del niño, influyen en la maduración de las partes del cerebro encargadas de la conciencia y regulación emocional (23), esto se confirma con algunas narrativas de los padres, como en la siguiente:

"En la forma de actuar, en la forma de expresarse, en la forma de convivir con otras personas o con uno mismo, o sea, uno se da de cuenta si el hijo está 
fallando o si está bien, porque si responde digamos agresivamente pues esto psicológicamente el hijo está mal de salud, o sea, algo le está fallando, puede ser que nosotros estemos fallando y que no sea él." (P2A1INT5).

\section{Autonomía}

Para el logro de la autonomía en los adolescentes, los padres que favorecen esta, contribuyen al desarrollo de la autoeficacia en sus hijos, ya que suelen usar prácticas educativas que promueven una mayor capacidad para pensar, formar opiniones propias y tomar decisiones, consiguiendo fomentar un mayor sentimiento de autoeficacia (24), en confirmación a lo anterior, los padres expresan las siguientes narrativas:

"Yo les digo a ellos, si los demás se quieren hacer lo que sea son ellos, ustedes no tienen que hacer lo mismo que ellos" (P7M4INT2).

"Pues yo creo que ellas son muy independientes, siempre han sido muy independientes desde muy niñas, casi siempre les ha tocado cuidarse solas, y pues la verdad ellas tratan de ser lo mejor en la casa y en el colegio" (P9M6INT1).

"Desde que he visto su desarrollo creo que es muy organizado en sus cosas, muy estudioso; todo, no le veo cosas malas a él, hablo de mi hijo" (P9P1INT6).

\section{Relaciones con los padres y vida familiar}

La relación con los padres y la vida familiar se constituyen (20) en el desarrollo socioafectivo del infante, ya que los modelos, valores, normas, roles y habilidades se aprenden durante el período de la infancia, la cual está relacionada con el manejo y resolución de conflictos, las habilidades sociales y adaptativas, con las conductas prosociales y con la regulación emocional. Los cambios en la familia, en las interacciones y en las pautas de crianza pueden afectar los estilos parentales y el desarrollo socioafectivo en

Tabla 5. Categorías definitivas

\begin{tabular}{|l|l|}
\hline $\begin{array}{l}\text { Categoría Definitiva } \\
\text { de Percepción de los } \\
\text { padres o acudientes }\end{array}$ & \multicolumn{1}{c|}{ Categoría Definitiva y aspectos que la componen } \\
\hline Bienestar Físico & $\begin{array}{l}\text { En esta categoría se incluyeron las narrativas que describían la necesidad de } \\
\text { determinar como prioridad la salud física en la calidad de vida de sus hijos, } \\
\text { a su vez, esta categoría incluye las expresiones que hacían relevancia en la } \\
\text { importancia de brindar a sus hijos una alimentación apropiada en base a una } \\
\text { dieta balanceada ya que esta determinará una muy buena salud. }\end{array}$ \\
\hline Bienestar & $\begin{array}{l}\text { En esta categoría se clasificaron las narrativas en donde los padres manifestaban } \\
\text { que la salud mental se encontraba asociada a la carga académica de sus hijos y } \\
\text { la forma en la que estos respondían a ella. }\end{array}$ \\
Psicológico & $\begin{array}{l}\text { Otra de las subcategorías que conforman esta categoría se orienta al estado } \\
\text { de ánimo y la forma en que se expresan frente al mismo con manifestaciones } \\
\text { de alegría, forma de hablar, mirar y vestir, actuar y convivir con los demás y } \\
\text { cómo siendo padres pueden reconocer dichos comportamientos y traducirlos en } \\
\text { "alertas", e incluso, reconocer si cursan por situaciones positivas o negativas } \\
\text { que puedan afectar su calidad de vida. }\end{array}$ \\
Estado de ánimo y & $\begin{array}{l}\text { Para esta categoría se incluyó un factor, que consideran los padres, afecta de } \\
\text { forma directa la calidad de vida de sus hijos: es el enamoramiento, en donde a } \\
\text { temprana edad se generan sentimientos derivados del noviazgo que repercuten en } \\
\text { el estado de ánimo llevándolos a emociones de tristeza, decepción y depresión, } \\
\text { e incluso a afectar el rendimiento académico y actividades cotidianas. }\end{array}$ \\
\hline
\end{tabular}




\begin{tabular}{|c|c|}
\hline Autonomía & $\begin{array}{l}\text { Esta categoría incluye las narrativas relacionadas con la toma de decisiones } \\
\text { por parte de sus hijos y cómo a través de los valores y formación en el hogar } \\
\text { ellos pueden reconocer cuándo se presenta una posible amenaza relacionada, } \\
\text { principalmente, con el consumo de drogas y la selección de amistades; a su vez, } \\
\text { en esta categoría se distingue la capacidad y el reconocimiento que otorgan los } \\
\text { padres a sus hijos mayores para delegar un rol sobre sus hermanos menores y } \\
\text { cómo este puede afectar la calidad de vida en sus hijos. Se incluyen, además, } \\
\text { las narrativas que permite identificar cómo los padres reconocen la disciplina } \\
\text { y la independencia que tienen sus hijos como algo positivo que promueve una } \\
\text { adecuada calidad de vida. }\end{array}$ \\
\hline $\begin{array}{l}\text { Relaciones con los } \\
\text { padres y vida familiar }\end{array}$ & $\begin{array}{l}\text { En esta categoría se incluyen las narrativas relacionadas con la vida familiar } \\
\text { y cómo resulta relevante la responsabilidad que tienen los padres de brindar } \\
\text { buen ejemplo a sus hijos con el fin de proporcionar un ambiente positivo para } \\
\text { los mismos. } \\
\text { Para esta categoría se incluyen las narrativas relacionadas con la dedicación } \\
\text { del tiempo que se brinda a los hijos y cómo en ocasiones, por cuestiones de } \\
\text { trabajo, se resta calidad al mismo, lo que repercute en el comportamiento y, por } \\
\text { consiguiente, afecta la salud y el bienestar de los adolescentes. } \\
\text { Se identifican las narrativas de los padres en cuanto al reconocimiento de } \\
\text { actitudes y comportamientos por parte de sus hijos que permite identificar } \\
\text { aspectos tanto negativos como positivos que repercuten en la calidad de vida } \\
\text { de los adolescentes. } \\
\text { Hacen parte de esta categoría las narrativas referentes a la sobrecarga que } \\
\text { identifican los padres al momento de asignar actividades al hermano mayor y el } \\
\text { cambio en el rol que surge por pasar de ser un hijo que requiere cuidado a ser } \\
\text { hermano que es cuidador. } \\
\text { Otro de los elementos tiene que ver con el apoyo emocional y la motivación que } \\
\text { brindan los padres y el reconocimiento por sus capacidades y habilidades, a su } \\
\text { vez, la identificación de las necesidades que surgen en ellos y cómo los padres } \\
\text { pueden reforzar los comportamientos positivos y reducir los comportamientos } \\
\text { negativos. }\end{array}$ \\
\hline $\begin{array}{l}\text { Apoyo social y relación } \\
\text { con los pares }\end{array}$ & $\begin{array}{l}\text { En esta categoría se incluyeron las subcategorías referentes a las relaciones } \\
\text { interpersonales de los adolescentes y su comportamiento en los diferentes } \\
\text { escenarios, principalmente en el ambiente escolar y cómo los amigos pueden } \\
\text { influenciar de forma positiva o negativa. A su vez, los padres identifican como } \\
\text { comportamiento precoz en el adolescente el inicio del enamoramiento. }\end{array}$ \\
\hline Ambiente escolar & $\begin{array}{l}\text { En esta categoría también se incluyeron las narrativas referentes a la relación } \\
\text { estrecha que existe cuando el adolescente se desarrolla en un ambiente familiar } \\
\text { inadecuado y cómo este repercute en el comportamiento con los pares y el } \\
\text { rendimiento académico. }\end{array}$ \\
\hline
\end{tabular}




\begin{tabular}{|l|l|}
\hline Acoso social (Bullying) & $\begin{array}{l}\text { En la subcategoría perteneciente a esta categoría, los padres reconocieron que } \\
\text { brindar confianza a sus hijos permite identificar si existen aspectos positivos o } \\
\text { negativos en el ambiente escolar que pueden influenciar en el comportamiento } \\
\text { del adolescente. }\end{array}$ \\
\hline Recursos económicos & $\begin{array}{l}\text { Otra de las subcategorías que conforman esta categoría es el cubrimiento de las } \\
\text { necesidades básicas por parte de los padres para sus hijos, y cómo estos deben } \\
\text { aprender a reconocer y valorar el esfuerzo por parte de los padres. }\end{array}$ \\
\hline Tiempo Libre - Ocio & $\begin{array}{l}\text { En esta categoría se clasificaron las subcategorías que hacían referencia a } \\
\text { las actividades que realiza el adolescente, como el deporte y la recreación en } \\
\text { familia como componentes que promueven la salud y el bienestar, contrario a } \\
\text { ello, los padres identificaron el uso inadecuado de la tecnología, el internet, y } \\
\text { las redes sociales como elementos que afectan directamente la calidad de vida. }\end{array}$ \\
\hline $\begin{array}{l}\text { Espiritualidad y Valores } \\
\text { Morales }\end{array}$ & $\begin{array}{l}\text { En la subcategoría perteneciente a esta categoría se incluyó el enfoque espiritual } \\
\text { y la formación en valores morales que brindan los padres a sus hijos, y cómo } \\
\text { estos influyen positivamente en la calidad de vida. }\end{array}$ \\
\hline $\begin{array}{l}\text { Co m p o r t a m i e n t o } \\
\text { general }\end{array}$ & $\begin{array}{l}\text { En esta categoría se dejaron todas las narrativas que demuestran cómo los } \\
\text { padres reconocen que las relaciones interpersonales con los pares pueden influir } \\
\text { de forma negativa en el comportamiento de los adolescentes. }\end{array}$ \\
\hline
\end{tabular}

Fuente: Elaboración propia de los autores

tal periodo. Los problemas emocionales de los padres, como la depresión, influyen en los niños, niñas y adolescentes, y les generan modelos inadecuados o prácticas negativas para su desarrollo (20). En confirmación a lo anterior con base en las respuestas de los padres, se ejemplifica en las siguientes narrativas:

“...todo está en lo que estén manejando ahí dentro del hogar, de que sean cosas positivas que a ellos no le repercutan para que estén mal" (P1M2INT2).

“...ellos demuestran cuando están mal con su forma de vestir, con su forma de hablar, con su forma hasta de mirar, con sus acciones y con su genio, de una vez dice uno algo pasa y esa es la alerta roja que le decía yo que tiene que estar pendiente" (P9P1INT19).

“...un proceso de paciencia yo veo que si tiene que hacer cambios eso no es de un día para otro en lo cual hay que acompañarlos y tenerles paciencia, y la cantaleta es lo peor, a nadie le gusta que lo cantaleteen, que pereza, entonces ya no gritan, ya no pelean ya no son envidiosos ya hacen las cosas sin que uno los mande y estudian voluntariamente, ¿ve?, o sea se notan muchos cambios en ellos y eso hay que valorárselo y decirle, "si ve, que bien; lo felicito" y estarlo felicitando por las cosas buenas y no decirle "es que usted si es necio, es que usted si es desordenado" (P7M1INT4).

\section{Apoyo social y relación con los pares}

Las presiones socioculturales como como el apoyo social y las relaciones con los pares pueden tener influencia de impacto negativo en la CVRS de los adolescentes como resultado de una mala CVRS global (25); Esto se evidencia en la narrativa de los padres, como en el siguiente ejemplo,

"Puede ser que en algunos casos se dejan influenciar de los amigos, eso también, así uno en la casa los lleve encaminaditos bien y todo, pero en la casa son una cosa y en el colegio son otra cosa, por lo general eso es casi todos, eso se llega siempre su momento en que ellos a veces les siguen la cuerda a los amigos que para no quedar mal" (P8M4INT2).

\section{Ambiente escolar}

La relación positiva entre familia y escuela podría contribuir de un modo decisivo al desarrollo de un apego escolar de los estudiantes por sus colegios, y si además la escuela crea un clima positivo para acoger a las familias y estructuras que puedan involucrarlas, se 
formará una efectiva relación familia-escuela con sus consecuentes impactos en los desempeños escolares integrales, que van más allá de la adquisición de conocimientos (26). Lo anterior, se confirma con la narrativa de los padres, quienes se expresan de la siguiente manera:

“...cuando un niño se porta mal en el colegio o tiene malas notas le caen al muchacho, yo pienso que al que deben corregir es al papá o a los papás o a la persona encargada del muchacho, si el chico está actuando así es por algo del núcleo familiar, por algo de lo que él está viviendo" (P2M1INT2).

\section{Acoso social (Bullying)}

El bullying es una conducta agresiva repetitiva que busca dañar o incomodar a otra persona, basada en un desbalance de poder con consecuencias psicológicas y sociales negativas para la vida de niños y adolescentes. Las víctimas de bullying perciben peor CVRS, asociados con tristeza e inestabilidad emocional, en donde el apoyo de pares y maestros influye en la relación entre ser víctima de bullying y la satisfacción en la vida (27). Con lo anterior, los padres logran identificar el bullying en sus hijos, expresado en la siguiente narrativa:

"Yo les digo si usted me está cambiando es por cosas del colegio, ¿qué pasa?, cuénteme, ¿es que están molestando, es que algún compañero le está diciendo que haga esto?" (P7M4INT2).

\section{Recursos económicos}

Independientemente del estrato socioeconómico se encontró que los adolescentes identificados con el género masculino tienen mejores niveles de bienestar psicológico en comparación con las adolescentes identificadas con el género femenino (28), con respecto a lo anterior, los padres expresan la siguiente narrativa:

"Por parte de los papás que tengan las cosas básicas, lo necesario así no tengan muchos lujos ni nada que tengan estrictamente lo necesario para vivir" (P1M7INT1).

\section{Tiempo Libre - Ocio}

En la dimensión de tiempo libre y ocio los padres destacan la tecnología comoun aspecto negativo en la CVRS de sus hijos, dado que la posible dependencia o adicción a la tecnología puede asociarse al uso intensivo de dispositivos tecnológicos en un hogar hiperconectado. No obstante, es un temor difuso, ya que asumen que sus hijos no dejan de imitar y reproducir sus propios comportamientos y actitudes hacia la tecnología. En este sentido, son conscientes de la responsabilidad que implican sus propios comportamientos y actitudes, sobre todo en edades tempranas (29). Con respecto a la anterior, los padres expresan las siguientes narrativas:

“...los distrae, no le están prestando atención a la clase, sino al celular, y por uno que tenga celular, los otros van a querer ver lo que ella o él está haciendo, qué es lo que está viendo..." (P5M3INT3).

“...es que de aquí a mañana él se va a dar cuenta que abstenerse, o sea controlar una cosa tan simple como el Facebook, y lo pude utilizar mañana para algo más importante y no tan solo para tener amigos y malas mañas y malas bobadas, si no tener Facebook para lo que es, para el servicio que debe prestar de verdad, como una... para generar un contacto sano, o sea algo bonito, familiar o con amigo..." (P7P1INT18).

\section{Espiritualidad y Valores Morales}

Los padres desempeñan un rol importante en la adquisición y mantenimiento de las creencias y conductas espirituales y religiosas en sus hijos. Estudios han demostrado una correlación positiva fuerte entre los padres religiosos y la reducción de conductas de riesgo y actividades criminales de sus hijos. Las creencias religiosas pueden ser transmitidas de una generación a otra y ayudar en la promoción de conductas saludables mediante la proscripción de aquellas que no lo son o están en riesgo (30). Teniendo en cuenta lo anterior, los padres expresan las siguientes narrativas con respecto a la importancia de la espiritualidad en sus hijos:

“...lo espiritual, porque eso es muy fundamental también porque si yo tengo temor a Dios yo voy a ir bien, porque el temor le hace a uno hacer las cosas siempre bien y tener uno responsabilidad" (P1M2INT3).

"Los principios morales siempre tienen que estar por encima, creo yo, y que hace parte de la salud y el bienestar" (P1P1INT1). 


\section{Comportamiento general}

Debido a los profundos y complejos cambios que se presentan en la adolescencia, educar a los adolescentes se convierte en una situación que genera tensión para muchos padres, estos experimentan sentimientos de ineficacia e incompetencia en relación con el comportamiento de sus hijos (31). Dado lo anterior, los padres expresan los comportamientos de sus hijos en la siguiente narrativa:

"la forma en cómo ellos se comporten y las relaciones que tengas personales con los demás” (P3M6INT1).

\section{Concepto de salud y bienestar de los adolescentes:}

Desde las perspectivas de los padres a continuación se presentan algunas narrativas:

"No van a estar ellos psicológicamente bien de salud porque van a empezar a darse todos los problemas de rechazo, de egoísmo, de si entonces la salud, no solo hablo de la salud física, de la salud, salud de todo, sino que la persona esté bien en todo, emocional, físico, intelectual, en todos los aspectos que pueda tener uno como persona, en lo físico, deporte". P1M8INT2v

\section{Conclusiones}

El carácter cualitativo del estudio permitió identificar desde la perspectiva de los padres y acudientes la percepción que estos tienen sobre las situaciones o hechos personales, familiares o escolares que influyen en la CVRS de sus hijos adolescentes escolarizados y otros aspectos del cotidiano que pueden afectar a sus hijos en la adolescencia.

Abordar a los padres o acudientes permitió identificar otros aspectos de la salud y calidad de vida que pueden afectar a sus hijos en la adolescencia. Surgieron dimensiones que no son reportadas por instrumentos de calidad de vida relacionada con la salud, como es la espiritualidad y la tecnología.

El reconocimiento de las percepciones de las madres, los padres y acudientes sobre la salud y calidad de vida de sus hijos resulta de gran relevancia para tener en cuenta las distintas dimensiones que lo componen y los requerimientos que de esto se desprende para tener en cuenta en la construcción de políticas públicas relacionadas con diferentes componentes, como por ejemplo, el empoderamiento de las familias, la generación de estrategias de información, comunicación y educación en beneficio de los jóvenes. Por otra parte, también se identifica que se requiere de trabajo intersectorial, en especial entre el sector salud y educación para abordar la salud y calidad de vida de los niños y jóvenes en los ambientes escolares.

\section{Limitaciones}

Las percepciones que se lograron identificar corresponden a un solo grupo focal. Se presentaron dificultades para poder lograr que los padres de familia fueran partícipes del grupo focal, pues, aunque se realizó la invitación a 20 padres de familia, solo se obtuvo respuesta efectiva de nueve, con los cuales se constituyó el grupo focal.

Existen pocos estudios cualitativos en la literatura desde la disciplina de enfermería sobre la CVRS de niños y adolescentes, desde la perspectiva de los padres que permitan cotejar la información de este estudio con otros estudios realizados.

\section{Referencias}

1. Martínez AJ, Serrate PC, Pacheco CL, Trasancos HD. Consideraciones sobre el concepto de salud y su enfoque en relación a la salud bucal. Rev Méd Electrón [Revista en Internet]. 2014 [Consultado 15 noviembre de 2016]; 36(5): 671- 680 "Disponible en: http://scielo.sld.cu/pdf/rme/v36n5/rme150514. pdf“"

2. Organización Mundial de La Salud;OMS [Documento en Internet]. 2016 [Actualizada 2016; consultado 15 noviembre 2016). "Disponible en: http:// www.who.int/features/factfiles/mental health/es/."

3. Quintero MC. Tres concepciones históricas del proceso salud-enfermedad. Hacia la Promoción de la Salud. [Revista Internet] 2007 [16 noviembre 2016]; 12: 41-50. "Disponible en: http://www.scielo.org.co/pdf/hpsal/v12n1/v12n1a03.pdf."

4. Jasso GL. Ortega LM. El impacto de los determinantes sociales de la salud en los niños. Boletín médico del Hospital Infantil de México. [Revista Internet] 2014[Consultado el 16 noviembre 2016]; 71(2. "Disponible en: http:// www.scielo.org.mx/scielo.php?script $=$ sci arttext\&pid=S1665-11462014000200009."

5. Barbero HA, Mesar LR, Azcona CS. Parent and child perception about the health related quality of life by weight status at Navarra, Spain. Arch Ar- 
gent Pediatr. [Revista Internet] 2013 [Consultado 15 noviembre 2016]; 111(6): 508-515. "Disponible en: $\quad$ http://www.scielo.org.ar/pdf/aap/v111n6/ v111n6a09.pdf

6. Maddaleno M, Morello P, Infante EF. Salud y desarrollo de adolescentes y jóvenes en Latinoamérica y El Caribe: desafíos para la próxima década. Salud pública de México [Revista Internet]2003 [Consultado 15 noviembre 2016]; 45(1):132.

7. Agudelo LS. Villa GA, Nieto RV. Percepción del estado de salud en la región central colombiana: Encuesta Nacional de salud. Rev. Salud Pública. [Revista Internet] 2012 [consultado el 17 noviembre 2016]; 14(6): 899-911. "Disponible en: http://www. scielosp.org/pdf/rsap/v14n6/v14n6a01.pdf."

8. Rajmil L, Roizen M, Urzúa A. Calidad de Vida y Salud en la Infancia y la Adolescencia. Rev. Tipica, Boletín Electrónico de Salud Escolar. [Revista Internet] 2010 [consultado 15 noviembre 2016]; 6 (2): 244-249. "Disponible en: https://www.researchgate. net/profile/Alfonso Urzua/publication/236904869 Calidad de Vida y salud en la infancia y la adolescencia/links/53f33f9d0cf2dd48950c9e27. pdf."

9. Quiceno J, Vinaccia S. Calidad de vida relacionada con la salud infantil: una aproximación conceptual. Rev. Psicología y Salud. [Revista Internet] 2008 [consultado el 15 noviembre del 2016]; 18 (1): 3744. "Disponible en: http://revistas.uv.mx/index.php/ psicysalud/article/view/673/1184."”

10. Deutsch kidscreen.org. [acceso marzo de 2017]. Disponible en: http://www.kidscreen.org/

11. Quiceno JM, Vinanccia S. Calidad de vida relacionada con la salud infantil: una aproximación desde la enfermedad crónica. Psychologia: avances de la disciplina. [Revista Internet] 2013 [Consultado 16 noviembre 2016]; 7(2): 69-86. "Disponible en: http://www.scielo.org.co/pdf/psych/ v7n2/v7n2a06.pdf."

12. Fernández JA, Fernández FM, Cieza A, Ravens SU. Medición de la calidad de vida en niños y adolescentes: comprobación preliminar de la validez y fiabilidad de la versión española del cuestionario KINDL. Atención Primaria [Revista Internet] 2004 [Consultado 15 noviembre 2016]; 33(8):434-442.

13. PedsQL TM. Pediatric Quality of Life Inventory TM. [acceso marzo de 2017]. Disponible en: http:// www.pedsql.org/

14. Health Act CHQ. CHQ: Child Health Questionnaire. [Acceso marzo de 2017]. Disponible en: http://www.healthact.com/chq.php

15. Deutsch kindl.org. [acceso marzo de 2017]. Disponible en: http://kindl.org/
16. Vilagut G, Ferrer M, Rajmil L, Rebollo P, Permanyer-Miralda G, Quintana JM, et al. The Spanish version of the Short Form 36 Health Survey: a decade of experience and new developments. Gac Sanit SESPAS. [Revista Internet] 2005 [Consultado 16 noviembre 2016]; 19(2): 135-150

17. Vélez CM, Lugo LH, García HI. Validez y confiabilidad del 'Cuestionario de calidad de vida KIDSCREEN-27' versión padres, en Medellín, Colombia. rev.colomb.psiquiatr. [Internet]. 2012[cited 2016 Nov 18]; 41(3): 588-605. Available from: http://www.scielo.org.co/scielo.php?script=Sci arttext\&pid=S0034-74502012000300010\&1ng=en.

18. Vélez CM, García HI. Medición de la calidad de vida en niños. Iatreia [Internet]. 2012 [cited 2017 Mar 22]; 25(3): 240-249. Available from: http:// www.scielo.org.co/scielo.php?script $=$ sci arttext\&pid=S0121-07932012000300007\&lng=en.

19. Buss Thofehrn Maira, López Montesinos Maria José, Rutz Porto Adrize, Coelho Amestoy Simone, Oliveira Arrieira Isabel Cristina de Mikla Marzena. Grupo focal: una técnica de recogida de datos en investigaciones cualitativas. Index Enferm [Internet]. 2013 Jun [citado 2016 Nov 10]; 22(1-2): 75-78. Disponible en: http:// scielo.isciii.es/scielo.php?script $=$ sci arttext\&pid=S1132-12962013001100116\&lng=es.

20. Jiménez GE, Aguilar CJ, García CG, López GP, Ferre JA, López CA, Hita OE. Influence of family environment of the development of obesity and overweight in a population of school children in Granada (Spain). Nutrición Hospitalaria [Articulo Internet]. 2012 [consultado 1 mayo 2017]; 27(1). "Disponible en: http://scielo.isciii.es/scielo.php?script=sci arttext\&pid=S0212-16112012000100021."

21. Vázquez N, Molina MC, Ramios P, Artazcoz L. Validación de un instrumento en español para medir habilidades parentales promovidas en una intervención de educación parental. Universidad de Barcelona [Revista Internet] 2016 [Citado 2 mayo 2017; 9(2)." Disponible en: http://revistes.ub.edu/index.php/ REIRE/article/viewFile/reire2016.9.2923/19218."

22. Ramírez LA, ferrando M, Gómez SM. ¿Influyen los estilos parentales y la inteligencia emocional de los padres en el desarrollo emocional de sus hijos escolarizados en $2^{\circ}$ ciclo de educación infantil? Acción psicológica [Revista internet] 2015 [citado 1 mayo 2017]; 12(1). "Disponible en. Http://revistas.uned.es/index.php/accionpsicologica/article/ view/14314/1350."

23. MC, Parra A, Oliva A. Percepciones de autoevaluación: Autoestima, autoeficacia y satisfacción vital 
en la adolescencia. Psychology, society y education [Revista Internet] 2010 [citado 1 mayo 2017]; 2(1). "Disponible en: http://repositorio.ual.es/bitstream/ handle/10835/2913/Reina\%20et\%20Al.pdf?sequence $=1 . "$

24. Cuervo MA. Pautas de crianza y desarrollo socioafectivo en la infancia. Diversitas: Perspectivas en Psicología. [Revista Internet] 2012 [Consultado 2 Junio 2017]; 6(1), 111-121. "Disponible en: http:// www.scielo.org.co/scielo.php?script $=$ sci arttext\&pid=S179499982010000100009\&lng=en\&tlng=es."

25. Kaartina S, Chin SY, Wahoda RF, Woon FC, Hiew CC, Zalilah MS, Nasir MT. Adolescent self-report and parent proxy-report of health-related quality of life: an analysisi of validity and reliability of pedsQL 4.o among a simple of malasyian adolescents and their parents. Health qual life outcomes. [Revista Internet] 2015 [citado el 1 mayo 2017]; 13:44. "Disponible en: https://www.ncbi.nlm.nih. gov/pmc/articles/PMC4397708/\#CR45."

26. Pizarro P Santana A, Vial B. La participación de la familia y su vinculación en los procesos de aprendizaje de los niños y niñas en contextos escolares. Diversitas: Perspectivas en Psicología. [Revista Internet] 2013 [consultado el 2 junio 2017]; 9(2), 271-287. "Disponible en: http:// www.scielo.org.co/scielo.php?script $=$ sci arttex-

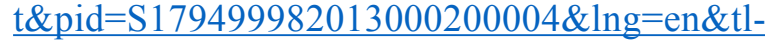
ng=es."

27. Hidalgo C, Molina T, Molina R, Sepúlveda R, Martínez V, Montaño R, et. Bullying y calidad de vida relacionada con la salud en adolescentes escolares chilenos. Rev. méd. Chile [Internet]. 2015 Jun [citado 2017 Jun 01]; 143(6): 716-723. Disponible en: $\quad$ http://www.scielo.cl/scielo.php?script=sci arttext\&pid=S0034-98872015000600004\&1ng=es. http://dx.doi.org/10.4067/S003498872015000600004.

28. Quiceno J., Vinaccia S., Calidad de vida, fortalezas personales, depresión y estrés en adolescentes según sexo y estrato, International Journal of Psychology and Psychological Therapy. [Revista Internet] 2014 [consultado 2 junio 2017]; 14(2): 155-170." Disponible en: http://www.ijpsy.com/volumen14/ num $2 / 381 /$ calidad-de-vida-fortalezas-personales-ES.pdf."

29. Lacave TT. Barrio TV, Barandalla LM. Percepción de los padres sobre el empoderamiento digital de las familias en hogares hiperconectados. [Internet] 2017 [citado 2 mayo 2017] 2(1)." Disponible en: http://www.elprofesionaldelainformacion.com/contenidos/2017/ene/10.pdf

30. Morales AL. Espiritualidad y Religión: percepciones de un grupo de Adolescentes puertorriqueños/as sobre su influencia en las conductas de Riesgo de la salud. Ciencias de la conducta [Internet] 2012 [citado 2 mayo 2017] 27 (1) 103-127. "Disponible en: http://www.albizu.edu/Portals/0/Documents/cau/ sju/Revista/2012/7_Religiosidad_Conductas de Riesgo.pdf."

31. Valenzuela Mujica María Teresa, Ibarra R. Ana María, Zubarew G. Tamara, Correa M. Loreto. Prevención de conductas de riesgo en el Adolescente: rol de familia. Index Enferm [Internet]. 2013 Jun [citado 2017 Jun 03]; 22(1-2): 50-54. "Disponible en: http://scielo.isciii.es/scielo.php?script $=$ sci arttext\&pid $=$ S1132-12962013000100011\&lng $=$ es. http:// dx.doi.org/10.4321/S1132-12962013000100011." 